\title{
A study of febrile seizures in children presenting to Teaching Hospital, Kurunegala
}

S P Pathirage ${ }^{1}$

Sri Lanka Journal of Child Health, 2010; 39: 47-48

(Key words: Febrile seizures, children, teaching hospital)

\begin{abstract}
Objective To describe demographic \& clinical aspects of febrile seizures (FS) presenting to Teaching Hospital (TH) Kurunegala

Method A prospective study was carried out on all children aged 1 month to 5 years, admitted to TH Kurunegala with a diagnosis of FS from February to November 2005. Children with symptomatic seizures, seizures associated with central nervous system infection and those who have had neonatal seizures were excluded. Data was obtained from medical records and direct interviewing of parents /guardians using a pretested questionnaire.
\end{abstract}

Results There were 2117 children aged 1 month to 5 years, admitted to TH Kurunegala during the study period. Of them $117(5.5 \%)$ had FS. Male to female ratio was 1.7:1. Sixty percent had no previous FS. Mean age at presentation was 17 months. Thirty two percent had their first seizure under the age of 1 year. Seventy four percent of FS occurred within the first 24 hours of onset of fever. Thirty four percent had complex FS. Around 10\% had developmental delay. A positive family history was obtained in $37 \%$ of patients. Febrile convulsive status was observed in 4 (3.4\%) children. Nine $(7.7 \%)$ children were on long term anticonvulsants.

\section{Introduction}

There are two published definitions of febrile seizures (FS). The International League Against Epilepsy (ILAE) defines a febrile seizure as "a seizure occurring in childhood after one month of age, associated with a febrile illness not caused by an infection of the central nervous system, without previous neonatal seizures or a previous unprovoked seizure, and not meeting criteria for other acute

${ }^{1}$ Senior Registrar in Paediatrics, Medical Intensive Care Unit, Lady Ridgeway Hospital, Colombo

(Received on 17April 2009. Accepted after revision on 26 June 2009) symptomatic seizures"1. The consensus statement published by the National Institute of Health (NIH) is similar to the ILAE definition, except for a lower age limit in the latter, when compared to the NIH lower age limit of 3 months ${ }^{1}$. Both definitions do not prevent children with developmental delay or neurological impairment being included under FS. FS recur in about $33 \%$ and about $2-4 \%$ will develop at least one unprovoked seizure ${ }^{1}$. A survey of febrile seizures at the Lady Ridgeway Hospital (LRH), Colombo was published in $2005^{2}$.

\section{Method}

All children admitted to a paediatric unit, Teaching Hospital (TH) Kurunegala with a diagnosis of FS from $1^{\text {st }}$ February 2005 to $30^{\text {th }}$ November 2005 were included in the study. Data was collected using a pretested questionnaire administered to the parents or guardians of these children. Exclusion criteria included children less than 1 month of age, those with central nervous system (CNS) infections and those with symptomatic seizures (e.g. due to hypoglycaemia). Patients with developmental delay were not excluded.

A simple $F S$ was defined as a generalized seizure with no focal seizure activity or focal manifestations during the postictal period, lasting less than 15 minutes and not recurring within the next 24 hours or during the same febrile illness.

A complex FS was defined as a seizure with features suggestive of focal activity at the onset, during or in the postictal period or a seizure lasting more than 15 minutes or a seizure that recurred within 24 hours or within the same febrile illness.

\section{Results}

Two thousand one hundred and seventeen children between the ages of one month and 5 years were admitted to $\mathrm{TH}$ Kurunegala from February to November 2005. One hundred and seventeen (5.5\%) of these children had FS. Male to female ratio was 1.7:1 with a mean age at presentation of 17 months. 
In $34 \%$ of patients the first seizure occurred under the age of one year and in $74 \%$, the seizures occurred within the first 24 hours of onset of fever. Thirty four percent had complex FS. A family history of FS in a first degree relative was obtained in $43(37 \%)$ patients. A family history of epilepsy was not present in any of the patients. Developmental delay, defined as a developmental score of less than $2 \mathrm{SD}$ or $70 \%$ for age, was present in $12(10 \%)$ patients. Two of these patients had global developmental delay (delay in 2 or more than 2 domains of development) ${ }^{3}$. Forty seven $(40 \%)$ patients presented with recurrences during the study period.

Nine $(8 \%)$ children were on long term anticonvulsant drugs viz. sodium valproate (4), phenobarbitone (3), clobazam (1) and carbamazepine (1). Febrile status epilepticus, defined as seizure activity lasting for more than 30 minutes, or 2 or more seizures occurring during a period of 30 minutes without the patient regaining consciousness in between, was observed in $4(3.4 \%)$ patients.

\section{Discussion}

The incidence of FS varies from $2-4 \%$ in Western Europe and USA, $5-10 \%$ in India, $8.8 \%$ in Japan and $14 \%$ in Guam ${ }^{1}$. However, the exact incidence of FS in Sri Lanka is not known. In my hospital based series $5.5 \%$ of children between the ages of one month and five years admitted to the paediatric unit had FS compared to $3 \%$ in the LRH series ${ }^{2}$. The incidence of complex FS in the study group was 34\% compared with $25 \%$ in the LRH series ${ }^{2}$. However, it should be noted that children with developmental delay were excluded from the LRH series ${ }^{2}$.

In $34 \%$ of patients in my series the first seizure occurred under the age of one year compared with $39 \%$ in the LRH series ${ }^{2}$. A family history of FS in a first degree relative was present in $37 \%$ of patients in my study compared with $25 \%$ in the LRH survey ${ }^{2}$.

The recurrence risk of FS in this study was found to be significantly higher among patients who have had a $1^{\text {st }}$ degree relative with FS and this observation correlates well with the findings of other workers ${ }^{1}$.
Eight percent of children were on long term anticonvulsants drugs. Whilst sodium valproate and phenorbarbitone are effective in prevention of FS recurrences, both agents have known adverse effects and cannot be recommended for prevention of febrile seizures ${ }^{4}$. However, clobazam and carbamazepine have no place in prophylaxis of $\mathrm{FS}^{4}$.

Some of the children in this study may have had epileptic seizures precipitated by fever. However, the exact number of patients that went on to develop epilepsy in this series is not known as the study was conducted over a period of 10 months.

\section{Acknowledgements}

I wish to acknowledge the help given by the supervising consultant Dr. Nimal Katugaha in conducting this study and the medical and nursing staff of ward 18, Teaching Hospital, Kurunegala for helping me throughout this study.

\section{References}

1. Waruiru C, Appleton R. Febrile Seizures: an update. Archives of Disease in Childhood 2004; 89(8):751-6.

2. Liyanage G, de Silva T U N, Perera B J C. A survey of febrile seizures at the Lady Ridgeway Hospital for Children. Sri Lanka Journal of Child Health; 2005; 34: 109 -13.

3. First LR, Palfrey JS. The infant or young child with developmental delay. New England Journal of Medicine 1994; 330 (7):478-83.

4. Rantala H, Tarkka R, Uhari M. A meta-analytic review of the preventive treatment of recurrences of febrile seizures, Journal of Paediatrics, 1997; 131(6): 922-5. 
